\title{
BUT OPENSAT 2019 SPEECH RECOGNITION SYSTEM
}

\author{
Martin Karafiát, Murali Karthick Baskar, Igor Szöke, Hari Krishna Vydana, Karel Veselý, Jan “Honza” Černocký \\ Brno University of Technology Speech@FIT and IT4I Center of Excellence \\ Brno, Czechia
}

\begin{abstract}
The paper describes the BUT Automatic Speech Recognition (ASR) systems submitted for OpenSAT evaluations under two domain categories such as low resourced languages and public safety communications. The first was challenging due to lack of training data, therefore various architectures and multilingual approaches were employed. The combination led to superior performance. The second domain was challenging due to recording in extreme conditions such as specific channel, speaker under stress and high levels of noise. Data augmentation process was inevitable to get reasonably good performance.
\end{abstract}

Index Terms - speech recognition, multilingual training, data augmentation, robustness

\section{INTRODUCTION}

The paper focuses on our work on building the ASR system for the Speech Analytic Technologies (OpenSAT) evaluation running in summer 2019 an organized by National Institute of Standards and Technology (NIST). It focused on three tasks: Automatic Speech Recognition (ASR), Speech Activity Detection (SAD) and Keyword Search (KWS) and on three domains: Low resourced languages (Babel), speech from video - (VAST, with SAD task only) and Public Safety Communications (PSC).

Our team worked on the ASR task for the following domains: Low resourced languages and public safety communications. While the first one is challenging by limited resources, the second one is addressing English but contains a lot of speech under stress and recorded through specific channel. To be successful in both challenges, several acoustic models were built (section 2). It is known that ASR systems tend to produce system-dependent errors, therefore complementarity and system fusion is crucial for good performance. Consequently, we focused on building more systems which vary in many parts to increase their complementarity: different acoustic units, training data (monolingual/multilingual, clean/noise), architectures (with/without convolution layers), acoustic modeling approaches (hybrid/end-to-end) and bandwidth (sampling frequency $8 / 16 \mathrm{kHz}$ ).

The detailed system descriptions are given in sections 3 and 4 and results are presented in sections 3.3 and 4.3

The work was supported by Czech Ministry of Education, Youth and Sports from the National Programme of Sustainability (NPU II) project "IT4Innovations excellence in science - LQ1602" and by the Office of the Director of National Intelligence (ODNI), Intelligence Advanced Research Projects Activity (IARPA) MATERIAL program, via Air Force Research Laboratory (AFRL) contract \# FA8650-17-C-9118. The views and conclusions contained herein are those of the authors and should not be interpreted as necessarily representing the official policies, either expressed or implied, of ODNI, IARPA, AFRL or the U.S. Government

\subsection{Low Resource Languages}

Pashto language was selected for this challenge from IARPA Babel program (running from 2012 to 2015) [1], therefore this challenge is later noted as Babel. In Babel, data from various low-resource languages were collected, which allowed us to focus on multilingual experiments and acoustic modeling. It is very natural for humans to borrow information from other sources when trying to learn a new language. Humans share the same vocal tract architecture and phonetic systems overlap across many languages, therefore automatic systems should be able to have components built and trained on various data sources.

During the Babel project, where we worked as part of "Babelon" team (led by $\mathrm{BBN}$ ), we verified that multilingual pre-training for feature extraction is an important technique especially if not enough training data is available [2]. Recently, we also extended multilingual Deep Neural Network (DNN) acoustics models [3] to novel NN architectures [4] and presented significant gains with adding more languages into acoustic model pre-training.

Consequently, we focused on system fusion of monolingual and multilingual models as we expected complementarity from various training data. Moreover, we built the systems with various architectures and modeling units, see 3.2 for details.

\subsection{Public Safety Communications}

On contrary to the pilot OpenSAT evaluations conducted in 2017 [5], where the target data was real fireman-dispatcher communication from the Charleston Sofa Super Store Fire in 2007, for OpenSAT 2019, NIST prepared simulated public safety communications collection ("SAFE-T") specifically designed for speech analytic systems. The data is intended to simulate a combination of characteristics found in public safety communications: background noises, channel transmission noises and speaking characteristics such as stress or sense of urgency.

In order to utilize additional corpora, like clean telephone data, we also experimented with simulated channel (see section 2.6 attempting to close the gap between target and clean training data. Finally, we focused on system fusion of various models trained only on "SAFE-T" data as well as models transferred from clean to target domain.

\section{ACOUSTIC MODELS AND TECHNIQUES}

\subsection{Hybrid Acoustics models}

Various classical hybrid DNN-HMM (Hidden Markov Model) speech recognizers were trained in Kaldi toolkit [6]. We decided to select Factorized Time Delay NN (TDNN-F) based architectures [7] as we were consistently obtaining superior performance to recurrent $\mathrm{NN}$ architectures used in last OpenSAT evaluations [5]: 
- TDNN-F: 15 layers with 1536 neurons, with bottle-neck factorization to 60 dimension and with stride 3 .

- CNN-TDNN-F: Similar to TDNN-F above, only the first 6 layers are replaced by convolutional ones.

The feature extraction was based on high-resolution MFCC features (40dim) concatenated with 100 dimensional online ivectors [8]. Next, the features were subsampled by factor 3 and NNs were trained with Lattice Free MMI (LF-MMI) objective and bi-phone targets as suggested in [9]. Finally, the NN are further trained with sequence Minimum Bayes Risk sMBR objective [10].

In order to increase the variety of our systems, we experimented with different units as acoustic model training targets: In addition to the bi-phone NN targets, which were shown to be on-par with the classical tri-phone ones [9], we experimented with single-state context independent phonemes as the $\mathrm{NN}$ model training targets.

\subsection{Multilingual hybrid models}

In previous evaluations [4, 5] we were using block-softmax objective function [11] which is not yet implemented with LF-MMI training in Kaldi. Therefore, we chose a much simpler approach: The training language data (audio lists, transcriptions, pronunciation dictionaries...) are simply stacked together with added language indicator for phoneme names as suggested in [12]. This approach is ensuring non mixing of acoustic units across the languages. Then, the training of multilingual $\mathrm{NN}$ is same as monolingual one.

Procedure of porting the multilingual $\mathrm{NN}$ into the new language can be described in the following steps: (1) the final multilingual layer (stacked all language-dependent phoneme units) are stripped and replaced with a new layer specific to target-language with random initialization. (2) Learning rate of the rest of $\mathrm{NN}$ is scaled by 0.5 . (3) The whole NN is retrained by two epochs on the target language.

\subsection{Sequence-to-sequence model}

With increasing interest in Sequence-to-sequence (seq2seq) models [13] 14], we decide to experiment with them in order to obtain complementary approach to classical hybrid DNN-HMM.

Joint CTC/Attention [15] based sequence-to-sequence ASR system is used in this work. The pipeline contains an input layer with a 2D-convolution layer followed by 12 multi-head self-attention [16] encoder layers. The 2D convolution layer sub-samples the input by factor of 4. The encoded input is fed to 12 multi-head restricted self-attention decoder layers. The restricted self-attention [17] is enforced using a 1D convolution component to focus only the neighboring region.

\subsection{Voice Activity detection}

Voice Activity detection (VAD) system was based on feed-forward Neural Network with 2 outputs. Standard mel-filter bank features (15 coefficients) with F0 estimates ( 3 coefficients) [18] were taken as the input after cepstral mean and variance normalization. Based on the training data we trained two VAD systems

- VAD-Babel: trained on publicly available Babel data (see section 3.1 .

- VAD-Safe-T: trained on downsampled $(8 \mathrm{kHz})$ PSC corpus (see section 4.1.

\subsection{Score combination}

ROVER tool from SCTK packag€耳 is used to perform score combination as a fusion of system outputs. To improve Normalized Cross Entropy (NCE) scores, logistic regression model is built to perform calibration. System word confidence and the language model score is provided as input to the training of the logistic regression model.

\subsection{PSC data enhancement}

The following methods of data enhancement were applied on the clean training data:

1. Adding reverberation (all training data).

2. Adding environmental noise (all training data).

3. Passing through walkie-talkie "channel" (1/2 of the training data).

4. Passing through codecs (all training data).

The audio data were processed on a segment chunks level. We used BABEL-VAD for speech/non-speech segmentation. Next, we made segment chunks of length 5 seconds (or more) by grouping particular segments in sequence. The chunk boundary is an event to change (randomly) the augmentation parameters - impulse response for reverberation, additive environmental noise, and SNR level. The motivation is to enlarge the speaker and environment variability.

The other set of augmentation steps is performed on level of the whole recordings.

\subsubsection{Adding speech reverberation}

We selected 2684 impulse responses from ACE (452), AIR 1.4 (52), REVERB (104), RWCP (1921), ReverbDB (155) datasets heaving RT60 lower than 0.5 seconds.

\subsubsection{Adding environmental noise}

We used various noise sources:

- Sounds downloaded from freesound.org (463 minutes in total) consisting of: 1) 37 minutes of engine noises, 2) 330 minutes of airplane cabin noises, 3) 96 minutes of HAM radio static noises.

- Environmental empty room noises from ReverbDB dataset (1054 minutes in total)

We mixed the data with $4 \mathrm{x}$ freesound (1852 minutes) $+1 \mathrm{x}$ reverbDB (1054 minutes).

Next, each chunk of segments was corrupted by a single, randomly selected noise picked from pool of 1452 samples of freesound (463 minutes $\times 4=1852$ minutes) and 1054 samples of ReverbDB (1054 minutes $\times 1=1054$ minutes). The starting position of the noise segment was randomly selected. The target SNR was chosen randomly from the interval $1-8 \mathrm{~dB}$ (low) and $9-15 \mathrm{~dB}$ (mid).

\subsubsection{Walkie-talkie "channel"}

We tried to simulate the HW (radio station) and ambiance in the following way:

1. Normalization of the training audio to $0 \mathrm{~dB}$ gain.

2. Increasing gain from $0 \mathrm{~dB}$ to $20 \mathrm{~dB}$ to introduce clippings.

${ }^{1}$ http://www1.icsi.berkeley.edu/Speech/docs/sctk-1.2/sctk.htr 
3. Application of randomly selected high-pass filter with cut-of frequency of $300,600,1000$, or $1500 \mathrm{~Hz}$.

4. Application of the phaser effect with "sox" tool to simulate phase distortions.

\subsubsection{Codecs}

The fire departments in the U.S. use the proprietary AMBE codec in their Digital Mobile Radio (DMR). The source code of this codec is not available. As a replacement, we used the EU version of the TETRA codec to simulate the effects of signal coding. We assume that the TETRA codec has characteristics similar to the AMBE one. We added also AMR, AMR-WB, G.711, G.726, G.729, GSM-FR, GSM-EFR, MP3 codecs with various bitrates (lower values) and up to $6 \%$ frame drop rates if possible.

\section{BABEL SYSTEM DESCRIPTION}

\subsection{Data}

Multilingual acoustic models were pre-trained on 20 Babel Languages (all languages available from Linguistic Data Consorcium LDC: Cantonese, Pashto, Turkish, Tagalog, Vietnamese, Assamese, Bengali, Hait. Creole, Lao, Swahili, Georgian, Tamil, Zulu, Kurdish Kurmanji, Tok Pisin, Cebuano, Kazakh, Telugu, Lithuanian, Guarani. The monolingual system was trained only on provided Pashto data-set (cca 92 hours).

\begin{tabular}{|l|l|l|}
\hline Cantonese & LDC2016S02 & IARPA-babel101-v0.4c \\
Assamese & LDC2016S06 & IARPA-babel102b-v0.5a \\
Bengali & LDC2016S08 & IARPA-babel103b-v0.4b \\
Pashto & LDC2016S09 & IARPA-babel104b-v0.4Yb \\
Turkish & LDC2016S10 & IARPA-babel105-v0.6 \\
Georgian & LDC2016S12 & IARPA-babel404b-v1.0a \\
Tagalog & LDC2016S13 & IARPA-babel106-v0.2g \\
Vietnamese & LDC2017S01 & IARPA-babel107b-v0.7 \\
Haitian Creole & LDC2017S03 & IARPA-babel201b-v0.2b \\
Swahili & LDC2017S05 & IARPA-babel202b-v1.0d \\
Lao & LDC2017S08 & IARPA-babel203b-v3.1a \\
Tamil & LDC2017S13 & IARPA-babel204b-v1.1b \\
Zulu & LDC2017S19 & IARPA-babel206b-v0.1e \\
Kurmanji & LDC2017S22 & IARPA-babel205b-v1.0a \\
Tok Pisin & LDC2018S02 & IARPA-babel207b-v1.0e \\
Cebuano & LDC2018S07 & IARPA-babel301b-v2.0b \\
Kazakh & LDC2018S13 & IARPA-babel302b-v1.0a \\
Telugu & LDC2018S16 & IARPA-babel303b-v1.0a \\
Lithuanian & LDC2019S03 & IARPA-babel303b-v1.0a \\
Guarani & LDC2019S08 & IARPA-babel305b-v1.0c \\
\hline
\end{tabular}

Table 1. Used Languages for multilingual pre-training.

The test and evaluation data were processed by VAD-BABEL and decoded with standard 3-gram back-off ARPA language model trained on acoustic model training data transcriptions and on WEB data downloaded during Babel program [19].

\subsection{Acoustic models}

The following models were trained for final fusion:

- Baseline_CNN-TDNN-F: CNN-TDNN-F based system trained purely on Pashto data
Table 2. WER on development data.

\begin{tabular}{lc}
\hline System & WER[\%] \\
\hline Baseline & 39.7 \\
Baseline_phnout & 40.3 \\
Multilingual_CNN-TDNN-F & 39.1 \\
Multilingual_TDNN-F & 39.0 \\
\hline Fusion & $\mathbf{3 7 . 4}$ \\
\hline
\end{tabular}

- Baseline_CNN-TDNN-F_phnout: CNN-TDNN-F using phoneme target units

- Multilingual_CNN-TDNN-F: CNN-TDNN-F system pretrained on 20 languages and transferred to the target language.

- Multilingual_TDNN-F: TDNN-F system pre-trained on 20 languages and transferred to the target language.

\subsection{Results}

Table 2 presents performances on the development (dev) set. We observed tiny $(0.6 \%)$ gain from using multilingual acoustic model over the monolingual one. It is probably due to the sufficient amount of training data (92 hours). Phoneme based acoustic model shows only $0.6 \%$ degradation over bi-phone baseline so we deem it suitable system for fusion. Moreover, it shows strong modeling power of NN based system trained with LF-MMI, compared to old Gaussian Mixture models where at least (bi/tri)-phonemes were needed to get reasonable performance. The final fusion shows significant $2.3 \%$ gain over monolingual baseline. This fusion also performed well on the evaluation data, see 'Babel' part of figure1 to compare with other participants in the OpenSAT Evaluation.

\section{PSC SYSTEM DESCRIPTION}

\subsection{Data}

Various English data-sets were used for acoustic model training:

- FULL-NB-CLEAN (2240 hours): English Fisher1+2, Switchboard 1 Release 2, Call Home English, AMI and ICSImeetings. The wide-band corpora (AMI and ICSI) were downsampled to $8 \mathrm{kHz}$.

- FULL-NB-NOISE (2240 hours): FULL-NB-CLEAN set further processed by channel enhancement (see section 2.6).

- MEETING-WB (174 hours): Only wide-band (WB) parts of $F U L L-N B-C L E A N$ (AMI, ICSI) with original sampling rate $16 \mathrm{kHz}$.

- SAFE-T-NB (20.6 hours): Target SAFE-T corpora downsampled to $8 \mathrm{kHz}$.

- SAFE-T-WB (20.6 hours): Target SAFE-T corpora with the original $16 \mathrm{kHz}$ sampling rate.

For the testing, we used 3-gram Language Model based on SAFE-T transcriptions. In addition, we trained RNN-LM (with 5 layers: TDNN, LSTM, TDNN, LSTM, TDNN) on the same data. It was used for final lattice re-scoring. Note, we experimented with RNN-LM also for Babel domain without any gain. Therefore, this technique is used only in PSC domain. 
Table 3. PSC: comparison of various pre-training methods.

\begin{tabular}{lcc}
\hline Pre-training NN & Method & WER[\%] \\
\hline None (SAFE-T-NB only) & - & 17.0 \\
FULL-NB-CLEAN & model-transfer & 15.8 \\
FULL-NB-NOISE & model-transfer & 15.3 \\
FULL-NB-NOISE & fine-tuning & $\mathbf{1 4 . 2}$ \\
\hline
\end{tabular}

\subsection{Acoustics models}

Various pre-training methods: We experimented with two techniques for porting of models trained on additional corpora (FULLNB-NOISE, FULL-NB-CLEAN) data into target SAFE-T-NB one:

1. model-transfer: Last layer is rebuilt on target data in the same way as in language-transfer learning used in porting of multilingual NN (see section 2.2), just the training/target language is same.

2. fine-tuning: further model training (4 epochs) on target data with scaling of learning rate by factor 0.05 .

Table 3 presents comparison of these two approaches on LFMMI CNN-TDNN-F models. The development data were processed with VAD-Babel, no RNN-LM and no sMBR training was used for simplicity. It shows significant $1.2 \%$ gain from using additional training data sources. Using noised data gives another $0.5 \%$ improvement. Simple fine-tuning instead of mode-transfer is taking advantage from pre-trained last layer (in model transfer the last layer is rebuilt on target data with random initialization) by giving additional $1.2 \%$. Therefore, simpler fine-tuning was selected as model-transfer method for later wide-band experiments where $M E E T I N G-W B$ was utilized for the training (see section 4.3.

Next, additional effect of training with SMBR and RNN-LM lattice rescoring is presented in table 4 It shows tiny $0.2 \%$ improvement by sMBR and $0.8 \%$ additional gain by RNN-LM.

Table 4. PSC: effect sMBR and RNN-LM on fine-tuned FULL-NBNOISE models.

\begin{tabular}{lc}
\hline System & WER[\%] \\
\hline Initial (LF-MMI CNN-TDNN) & 14.2 \\
+ sMBR & 14.0 \\
+ RNN-LM & 13.2 \\
\hline
\end{tabular}

Sequence-to-sequence training Experiments with sequence-tosequence training were conducted using EspNET [20] toolkit. 83 dimensional log-Mel filter-bank features are used as input and 5002 sentence-piece units are used as output labels. The encoder and decoder contain 2048 dimensional hidden units and 4 attention heads. CTC weight of 0.3 is applied during training and decoding. The model training is regularized with dropout $(0.1)$ and label smoothing (0.1). Spectral augmentation is applied by time warping and frequency masking. The final model is obtained by averaging the best 10 models. The performance of the system is denoted in table 5 .

\subsection{Results}

The following models were used for final fusion:
- SAFE-T-NB CNN_TDNN-F baseline trained in 8kHz sampling sampling rate

- SAFE-T-WB CNN_TDNN-F baseline trained in 16kHz sampling sampling rate

- FULL-NB-NOISE: CNN_TDNN-F model pre-trained on $F U L L-N B$-NOISE data and further trained on SAFE-T-NB (3 epochs).

- MEETING-WB: CNN_TDNN-F model pre-trained on $M E E T I N G-W B$ data and further trained on SAFE-T-WB (3 epochs).

- Seq2seq-WB: Seq2seq model pretrained on 960 hours of Librispeech data and fine-tuned to $S A F E-T-W B$ data.

Table 5. PSC: final systems results.

\begin{tabular}{lcc}
\hline System & \multicolumn{2}{c}{ WER[\%] } \\
& VAD-Babel & VAD-SAFE-T \\
\hline SAFE-T-NB & 16.4 & 15.3 \\
SAFE-T-WB & 15.0 & (2) 14.0 \\
FULL-NB-NOISE & $(1) \mathbf{1 3 . 2}$ & 13.7 \\
MEETING-WB & 13.8 & 14.1 \\
Seq2seq-WB & $(3) 16.5$ & - \\
\hline fusion (1)+(2) & \multicolumn{2}{c}{12.4} \\
(no score calibration) & \multicolumn{2}{c}{12.2} \\
fusion (1)+(2) & \multicolumn{2}{c}{11.7} \\
fusion (1)+(2)+(3) & \multicolumn{2}{c}{11.1} \\
fusion all (no score calibration) & \multicolumn{2}{|c|}{$\mathbf{1 1 . 0}$} \\
fusion all & \multicolumn{2}{c}{} \\
\hline
\end{tabular}

Table 5 presents $1.3-1.4 \%$ absolute improvement using original WB data instead of downsampling to $8 \mathrm{kHz}$. Training with help of noised data $(8 \mathrm{kHz})$ is getting additional $0.3-1.8 \%$ gain over widespectra training and presents $1.6-3.2 \%$ improvement over $8 \mathrm{kHz}$ baseline. Therefore, FULL-NB-NOISE based system was our single best system. Unfortunately, we did not have time to create MEETING-WB-NOISE data-set, therefore we fine-tuned only clean MEETING-WB generating intermediate results.

Our systems in PSC section vary a lot, therefore we run a detailed analysis with focus on complementarity. The most complementary system to the best single system, mark as (1) in table 5 was SAFE-T-WB (2) processed by SAFE-VAD, probably due to different bandwidth and segmentation. End-2-End system performs close to hybrid baseline and helps in system fusion by additional $0.5 \%$ over $(1)+(2)$. Fusion of all systems provides $0.7 \%$ additional gain and shows $2.2 \%$ absolute improvement over the best single system. In addition, we present small $0.1-0.2 \%$ gain by score calibration. Finally, we got really good results on the eval data in comparison to the other evaluation participant (see PSC part of figure 1).

\section{CONCLUSION}

The paper presented our efforts for OpenSAT evaluation 2019. We participated in two domains: Low Resource Languages and Public Safety Communications. In the first one, we confirmed the importance of creating highly complementary systems as well as using multilingual approaches. In the second one, we described our specific training data enhancements and investigated complementarity with recent end-to-end based system. In both domains, our systems had the best performance in the OpenSAT Evaluations. 


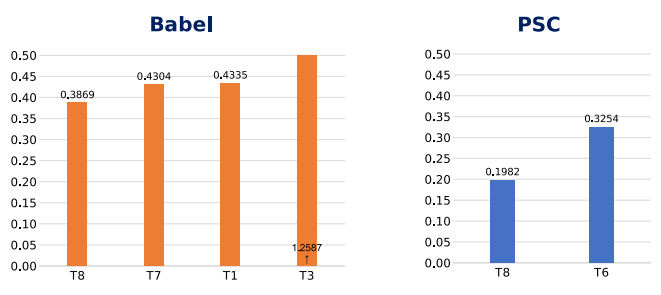

Fig. 1. Evaluation results in WER ("T8" is BUT system).

\section{REFERENCES}

[1] M. Harper, "The BABEL program and low resource speech technology," in Proc. of ASRU 2013, Dec 2013.

[2] Frantisek Grézl and Martin Karafiát, "Adapting multilingual neural network hierarchy to a new language," in Spoken Language Technologies for Under-Resourced Languages, 2014.

[3] Arnab Ghoshal, Pawel Swietojanski, and Steve Renals, "Multilingual training of deep neural networks.," in ICASSP. 2013, pp. 7319-7323, IEEE.

[4] Martin Karafiát, K. Murali Baskar, Pavel Matějka, Karel Veselý, František Grézl, Lukáš Burget, and Jan Černocký, "2016 BUT babel system: Multilingual BLSTM acoustic model with i-vector based adaptation," in Proceedings of Interspeech 2017, 2017.

[5] Martin Karafiát, K. Murali Baskar, Igor Szőke, Vladimír Malenovský, Karel Veselý, František Grézl, Lukáš Burget, and Jan Černocký, "But opensat 2017 speech recognition system," in Proceedings of Interspeech 2018. 2018, vol. 2018, pp. 26382642, International Speech Communication Association.

[6] Daniel Povey, Arnab Ghoshal, Gilles Boulianne, Lukas Burget, Ondrej Glembek, Nagendra Goel, Mirko Hannemann, Petr Motlicek, Yanmin Qian, Petr Schwarz, Jan Silovsky, Georg Stemmer, and Karel Vesely, "The kaldi speech recognition toolkit," in IEEE 2011 Workshop on Automatic Speech Recognition and Understanding. Dec. 2011, IEEE Signal Processing Society, IEEE Catalog No.: CFP11SRW-USB.

[7] Daniel Povey, Gaofeng Cheng, Yiming Wang, Ke Li, Hainan $\mathrm{Xu}$, Mahsa Yarmohammadi, and Sanjeev Khudanpur, "Semiorthogonal low-rank matrix factorization for deep neural networks," in Proceedings of Interspeech, 09 2018, pp. 37433747.

[8] Vijayaditya Peddinti, Guoguo Chen, Daniel Povey, and Sanjeev Khudanpur, "Reverberation robust acoustic modeling using i-vectors with time delay neural networks," in Proceedings of Interspeech, 2015.

[9] Daniel Povey, Vijayaditya Peddinti, Daniel Galvez, Pegah Ghahremani, Vimal Manohar, Xingyu Na, Yiming Wang, and Sanjeev Khudanpur, "Purely sequence-trained neural networks for asr based on lattice-free mmi," in Proceedings of Interspeech, 09 2016, pp. 2751-2755.

[10] Karel Veselý, Arnab Ghoshal, Lukáš Burget, and Daniel Povey, "Sequence-discriminative training of deep neural networks," in Proceedings of Interspeech 2013. 2013, pp. 2345-2349, International Speech Communication Association.
[11] Karel Veselý, Martin Karafiát, František Grézl, Miloš Janda, and Ekaterina Egorova, "The language-independent bottleneck features," in Proceedings of IEEE 2012 Workshop on Spoken Language Technology. 2012, pp. 336-341, IEEE Signal Processing Society.

[12] Francis Keith, William Hartmann, Man-Hung Siu, Jeff Ma, and O Kimball, "Optimizing multilingual knowledge transfer for time-delay neural networks with low-rank factorization," in Proc. ICASSP 2018. 04 2018, pp. 4924-4928, IEEE Signal Processing Society.

[13] Jan K Chorowski, Dzmitry Bahdanau, Dmitriy Serdyuk, Kyunghyun Cho, and Yoshua Bengio, "Attention-based models for speech recognition," in NIPS, 2015, pp. 577-585.

[14] Shinji Watanabe, Takaaki Hori, Suyoun Kim, John R Hershey, and Tomoki Hayashi, "Hybrid CTC/attention architecture for end-to-end speech recognition," IEEE Journal of Selected Topics in Signal Processing, vol. 11, no. 8, pp. 1240-1253, 2017.

[15] Shinji Watanabe, Takaaki Hori, Suyoun Kim, John R Hershey, and Tomoki Hayashi, "Hybrid CTC/attention architecture for end-to-end speech recognition," IEEE Journal of Selected Topics in Signal Processing, vol. 11, no. 8, pp. 1240-1253, 2017.

[16] Ashish Vaswani, Noam Shazeer, Niki Parmar, Jakob Uszkoreit, Llion Jones, Aidan N Gomez, Łukasz Kaiser, and Illia Polosukhin, "Attention is all you need," in Advances in neural information processing systems, 2017, pp. 5998-6008.

[17] Baosong Yang, Longyue Wang, Derek Wong, Lidia S. Chao, and Zhaopeng Tu, "Convolutional self-attention networks," 2019.

[18] Pegah Ghahremani, Bagher BabaAli, Daniel Povey, Korbinian Riedhammer, Jan Trmal, and Sanjeev Khudanpur, "A pitch extraction algorithm tuned for automatic speech recognition," in Acoustics, Speech and Signal Processing (ICASSP), 2014 IEEE International Conference on, Florence, Italy, May 2014, IEEE.

[19] Le Zhang, Damianos Karakos, William Hartmann, Roger Hsiao, Richard M. Schwartz, and Stavros Tsakalidis, "Enhancing low resource keyword spotting with automatically retrieved web documents," in INTERSPEECH 2015, 16th Annual Conference of the International Speech Communication Association, Dresden, Germany, September 6-10, 2015, 2015, pp. 839-843.

[20] Shinji Watanabe, Takaaki Hori, Shigeki Karita, Tomoki Hayashi, Jiro Nishitoba, Yuya Unno, Nelson Enrique Yalta Soplin, Jahn Heymann, Matthew Wiesner, Nanxin Chen, Adithya Renduchintala, and Tsubasa Ochiai, "Espnet: End-to-end speech processing toolkit," in Interspeech, 2018, pp. 22072211. 